\title{
Particle Mass Spectrometer (PMS) and its Application to Nano-Particle Sizing in Various Systems ${ }^{\dagger}$
}

\author{
C. Janzen, M.G.D. Strecker and P. Roth \\ Institut für Verbrennung und Gasdynamik \\ Gerhard-Mercator-Universität Duisburg*
}

\begin{abstract}
Particle mass spectrometry has been shown to be an effective diagnostic technique to determine particle size and size distribution of charged nano-particles originating from different reactive sources. In the present paper, the principle of the PMS is described in detail and examples of application to various particle formation systems are presented. A spark generator, a microwave reactor, and a low-pressure premixed flame burner were used to produce particles of different materials in different size ranges between 1 and $10 \mathrm{~nm}$. The resolution and sensitivity of the PMS has been improved by the adaptation of a preamplifier consisting of a secondary electron multiplier coupled to an external conversion dynode. The particle mass - being the main parameter of the measurements - is not influenced by the additional acceleration due to the conversion current. The use of a secondary electron multiplier leads to a signal gain of $10^{4}$ compared with measurements without the preamplifier.
\end{abstract}

\section{Introduction}

A large number of commercial powders, e.g. for the semiconductor industry, pigments, carbon blacks, and high-purity materials for optical fibers and thin films, are produced via aerosol routes, see for example [1] - [4]. The control of the process is necessary in order to determine the powder characteristics and to specifically influence the properties of the product particles, [5]. Consequently, in-situ analysis is desired to follow the formation process and to control the characteristics of the particles. Available techniques for sizing and detecting aerosol particles are often limited to operation near atmospheric pressure (e.g. differential mobility analyser, condensation nuclei counter, diffusion batteries, etc.). Furthermore, most of the methods require sampling and dilution prior to analysis [6], which may change the powder characteristics. It is thus necessary to realize a continuous sampling method which has no effect on the particle characteristics and which is effective in size determination.

Molecular beam sampling, which is widely known from conventional time-of-flight mass spectrometry, can fulfill the requirements of almost undisturbed, continuous sampling, thus giving a clear image of the reactive environment in the probe. Disturbances are avoided by an expansion into the free-molecular regime

* 47048 Duisburg, Germany

+ Received: May 17, 1999 with coinciding rapid freezing of the sample. The particle mass spectrometer described here [7] allows the determination of the particle size and size distribution by combining measured particle kinetic energy and particle velocity. In contrast to the particle beam mass spectrometer used by Ziemann et al. [8], the PMS does not require any calibration and is applicable for particles down to $1 \mathrm{~nm}$ and large molecules.

\section{Measurement Principles}

The basic principle of the particle beam mass spectrometer is the behavior of charged particles in an electric field. The situation is shown schematically in Fig. 1. The particle beam enters a uniform electric field in a capacitor of length $l_{C}$ and width $b_{C}$ supplied with a voltage $U_{C}$. The lines of force of the electric field are assumed to exist only inside the capacitor and are perpendicular to the electrode surfaces. The field strength therefore is $E=U_{C} / b_{C}$. An individual charged particle entering the capacitor is deflected by a force $F_{y}=z e U_{C} / b_{C}$. It causes an acceleration in the $\mathrm{y}$-direction in relation to its mass $\ddot{y}=z \cdot e \cdot U_{C} /\left(b_{C} \cdot m_{P}\right)$. The trajectories of the particles inside the capacitor can be described as follows:

$$
\begin{array}{ll}
y_{P}=\frac{1}{2} \cdot \frac{z \cdot e \cdot U_{C}}{m_{P} \cdot b_{C}} \cdot t^{2} & x_{P}=v_{P} \cdot t \\
\dot{y}_{P}=\frac{z \cdot e \cdot U_{C}}{m_{P} \cdot b_{C}} \cdot t & \dot{x}_{P}=v_{P} .
\end{array}
$$



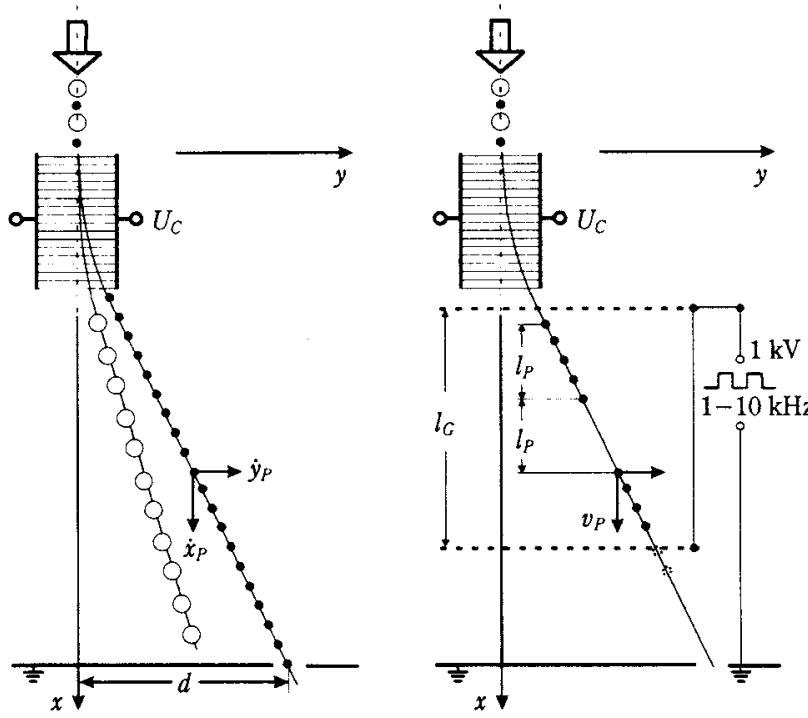

The particle mass $m_{P}$ can be determined from the deflection voltage $U_{C}$, if the velocity of the particles is known. The set-up shown on the right side of Fig. 1 is used to measure the required velocity of the particles. The selected beam of charged particles passes two grids supplied with a synchronously pulsed repelling potential. The upper grid forms packages of length $l_{P}$ which depend on the frequency $f$ of the grid voltage and the particle velocity $v_{P}$. Only very few or no particles can pass the second grid if the particle package length $l_{P}$ is an odd-numbered multiple of the distance $l_{G}$ between the grids. By changing the frequency of the deflection voltage $U_{C}$, a fluctuating particle current with maxima and minima is obtained. These minima and maxima of the particle current $I_{P}$ obey the following conditions:

$$
\begin{aligned}
& v_{P}=2 \cdot f_{\min , i} \cdot l_{p, i} \\
& l_{p, i}=l_{G}, l_{G} / 3, l_{G} / 5, \text { etc. }
\end{aligned}
$$
ages and operation of the electrical filter supplied with synchronously pulsed high voltage.

After leaving the capacitor, the particles move along straight lines as indicated on the left side of Fig. 1. Assuming constant velocity components $\dot{y}_{P}$ and $\dot{x}_{P}$, the trajectory of an individual particle is therefore given by:

$$
y_{P}=\frac{z \cdot e \cdot U_{C}}{m_{P} \cdot v_{P}^{2}} \cdot \frac{l_{C}}{b_{C}} \cdot\left(x_{P}-\frac{l_{C}}{2}\right) \quad \text { for } x_{P} \geq l_{C} .
$$

Since every particle of mass $m_{P}$ and charge $z$ has its individual trajectory, the originally uniform and wellfocused particle beam is split up into a fan-shaped beam. The mass classification is now realized by introducing a "monochromator" slit which selects particles of one distinct mass whereas the others are blocked. The slit is located at a distance of $x=l$ downstream of the capacitor, has a slit width $\Delta d$, and a position $y=d$ from the beam axis. By rearranging Eq. (2) and introducing $y_{P}=d$ and $x_{P}=l$, the kinetic energy selecting operation of the whole arrangement is:

$$
E_{k i n}=\frac{m_{P}}{z \cdot e} \cdot \frac{v_{P}^{2}}{2}=U_{C} \cdot \frac{l_{C}}{b_{C}} \cdot\left(\frac{2 \cdot l}{d}-\frac{l_{C}}{d}\right) .
$$

The equation above indicates that for known values of $l_{c}, b_{C}, d$, and $l$, the voltage $U_{C}$ selects particles of kinetic energies $m_{P} \cdot v_{P}^{2} / 2$ carrying $z$ elementary charges. By varying the deflection voltage $U_{C}$, particles of various kinetic energies can be made to pass the "monochromator" slit. Thus:

$$
U_{C}=\text { const. } \cdot \frac{m_{P}}{z \cdot e} \cdot \frac{v_{P}^{2}}{2} \text {. }
$$

Combination of Eqs. (4) and (5) leads to

$$
U_{C}=\text { const. } \cdot \frac{m_{P}}{z \cdot e} .
$$

The resultant signals are current/voltage spectra of the kinetic energy of the particles. From the principle of the particle mass spectrometer (PMS), it is also clear that the current/voltage spectra are not the probability density function (PDF) of the particle mass or the kinetic energy, because the sensitivity of the PMS depends on the deflection voltage. Roth and Hospital [9] have shown the following relation between the measured particle current, the deflection voltage $U_{C}$, and the PDF to be valid:

$$
\operatorname{PDF}\left(m_{P}\right)=\text { const. } \frac{I\left(U_{C}\right)}{U_{C}}
$$

The realization of the particle mass spectrometer is schematically shown in Fig. 2. It consists of a molecular beam sampling system by which a small sample taken from the respective aerosol generator is supersonically expanded through a silver-plated and electrically grounded glass nozzle $\boldsymbol{a}$ into a vacuum chamber where the pressure is kept at about $10^{-3}$ mbar. The supersonic free jet formed by the nozzle expansion flow contains both particles and gas molecules. The flow conditions are such that the gas temperature decreases very rapidly, freezing any physical and chemical reactions inside the expanding sample almost completely. The center of the free jet is extracted by a sharp-edged skimmer $\boldsymbol{b}$ and moves as a "particleloaded" molecular beam into the second vacuum chamber. The molecular beam is directed through a 


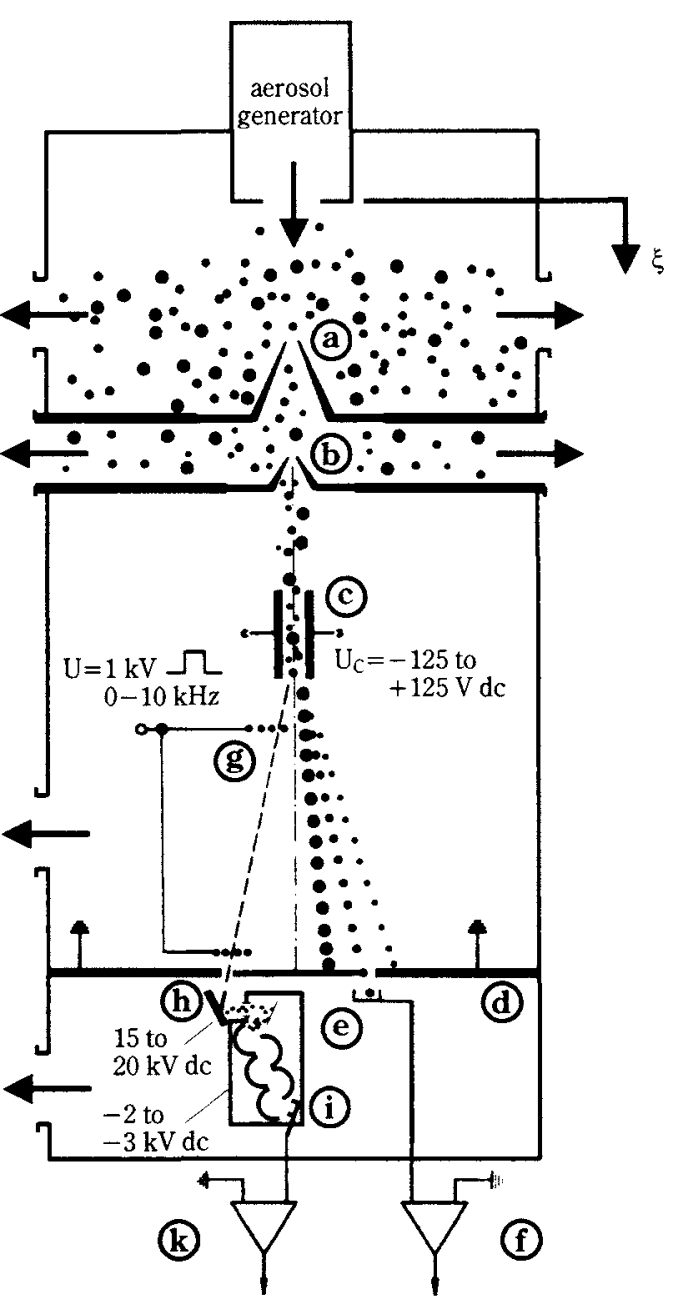

Fig. 2 Schematics of the PMS. $a$ nozzle, $b$ skimmer, $\boldsymbol{c}$ electrostatic deflection plates, $\boldsymbol{d}$ electrically grounded blend with slits, $e$ Faraday-Cup, $f$ electrometric amplifier, $g$ repelling grids, $\boldsymbol{h}$ conversion dynode, $\boldsymbol{i}$ secondary electron amplifier, $\boldsymbol{k}$ electrometric amplifier.

deflection capacitor $\boldsymbol{c}$, where charged particles are deflected from the beam axis and analysed due to their kinetic energy, as described above. The particles deliver their charge to a Faraday-Cup $\boldsymbol{e}$, which is connected to an ultra-sensitive amplifier $\boldsymbol{f}$.

In order to improve the sensitivity of the PMS, a third vacuum chamber $\boldsymbol{d}$ is added to the PMS. The pressure in this chamber was maintained at about $10^{-8}$ mbar by a turbomolecular pump in order to obtain a further signal amplification compared with the Faraday-Cup. It consists of a conversion dynode $\boldsymbol{h}$ and a secondary electron multiplier $i$. The dynode is supplied with a high positive voltage of 15 to $20 \mathrm{kV}$. This high electric field accelerates negatively charged particles from an initial kinetic energy of some hundred $\mathrm{eV}$ to some keV. The energy of the particles is then high enough to produce secondary electrons or ions when impacting on the surface of the dynode. The secondary charge carriers are further accelerated towards the cathode of the secondary electron multiplier which consists of 17 dynodes, causing an exponential electron increase by a factor of about $10^{6}$ to $10^{8}$. The generated current can be further amplified by an ultra-sensitive amplifier $\boldsymbol{k}$.

\section{Experimental Study and Results}

In this study, four different types of particle sources have been connected to the PMS, and the respective mass evolutions of the nano-sized particles have been studied in:

- premixed low-pressure $\mathrm{H}_{2} / \mathrm{O}_{2} / \mathrm{Ar}$ flames doped with $\mathrm{SiH}_{4}$ to produce $\mathrm{SiO}_{2}$ particles,

- a spark generator to produce charged particles at different spark frequencies $10 \mathrm{~Hz} \leq f \leq 1000 \mathrm{~Hz}$ from different materials,

- a microwave plasma burning in $\mathrm{C}_{2} \mathrm{H}_{2} / \mathrm{Ar}$ mixtures to generate nano-sized soot particles,

- premixed low-pressure $\mathrm{C}_{2} \mathrm{H}_{2} / \mathrm{O}_{2} / \mathrm{Ar}$ flames to produce soot particles.

In the case of soot particle formation in low-pressure hydrocarbon flames, Faraday-Cup measurements were compared with results obtained from secondary-electron multiplier measurements.

In the first series of experiments, the PMS was connected to a low-pressure flame reactor. Mixtures of hydrogen, oxygen, and argon were doped with small amounts of $\mathrm{SiH}_{4}$ to produce ultrafine silica particles [10]. The pressure was held between 25 and $30 \mathrm{mbar}$ providing an extended reaction and particle formation zone, thus ensuring a higher spatial resolution. The gases were premixed and supplied to the burner head by mass-flow controllers. The low-pressure burner was movable in the horizontal direction to vary the distance between the burner head and the probing nozzle, i.e., the flow coordinate $\xi$. Examples of simultaneously measured current/voltage spectra and current/frequency spectra of positively charged particles, from which the particle mass can be determined, are shown in Fig. 3. Samples containing silica particles were taken from the low-pressure burner at a flow coordinate of $\xi=60 \mathrm{~mm}$. The flame conditions are given in the figure caption. The upper part of Fig. 3 shows the particle current measured with the FaradayCup as a function of the deflection voltage. The curve shows one distinct maximum at $U_{C}=11 \mathrm{~V}$. According to Eq. (6), the deflection voltage can be directly related to the particle kinetic energy. For a singly charged particle, a voltage of $U_{C}=11 \mathrm{~V}$ results in a kinetic energy of $E_{k i n}=4.7 \cdot 10^{-17} \mathrm{~J}$. The measurement for the 

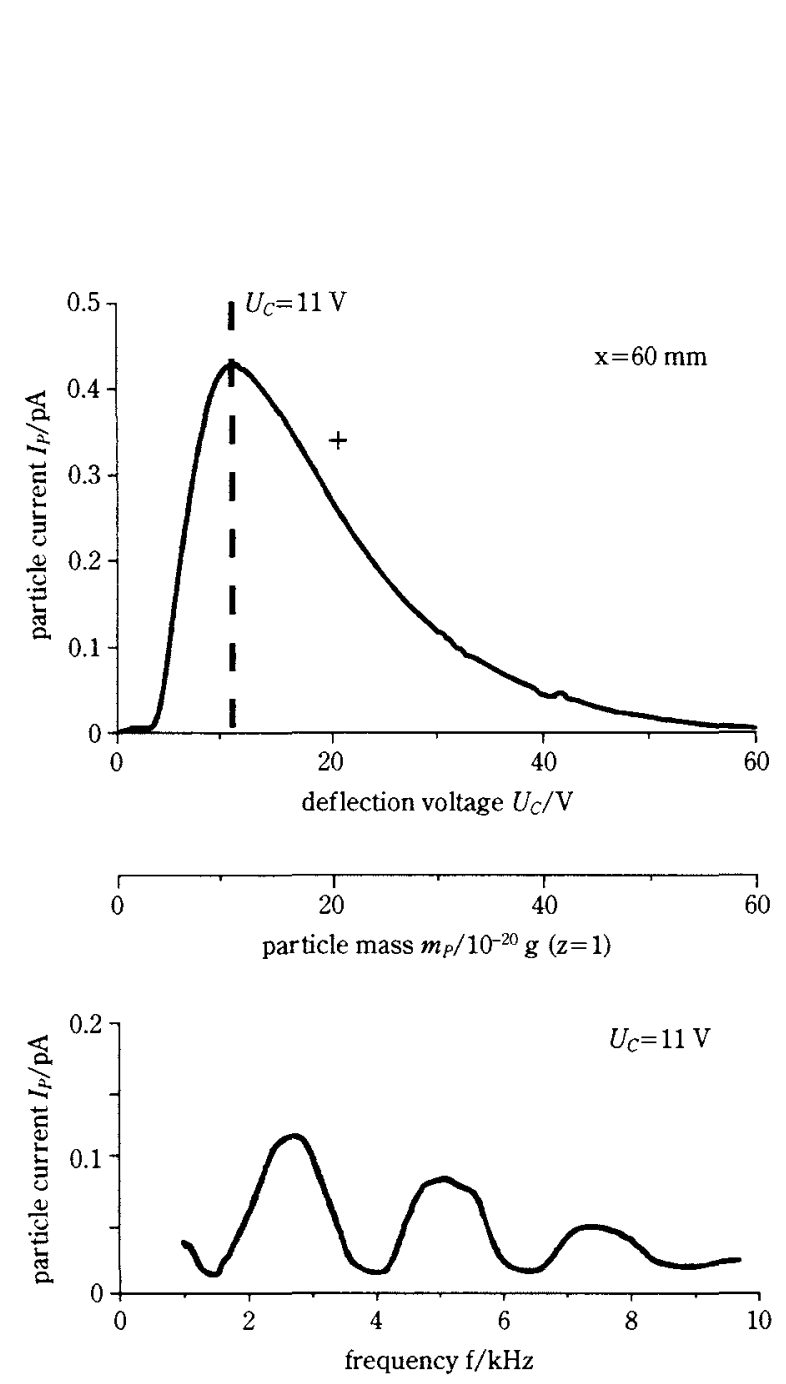

Fig. 3 Example of simultaneously measured kinetic energy and velocity of positively charged particles. Flame conditions: $\xi=60 \mathrm{~mm}, v_{u}=1.44 \mathrm{~m} \mathrm{~s}^{-1}, p=27.5 \mathrm{mbar}, \mathrm{H}_{2} / \mathrm{O}_{2}=1.69$, $\mathrm{Ar} /\left(\mathrm{H}_{2}+\mathrm{O}_{2}\right)=1.36,262 \mathrm{ppm} \mathrm{SiH}_{4}$.

determination of the particle velocity shown in the lower part of Fig. 3 was performed for particles deflected at $U_{C}=11 \mathrm{~V}$. The measured currents plotted as a function of the frequency of the repelling grid's voltage exhibit minima at $f_{\min 1}=1.3 \mathrm{kHz}, f_{\min 2}=3.9 \mathrm{kHz}$, and $f_{\min 3}=6.5 \mathrm{kHz}$. Applying Eq. (5) leads to a particle velocity of about $650 \mathrm{~m} \mathrm{~s}^{-1}$. Combination of Eq. (4) and (5) results in a mean particle mass of $22 \cdot 10^{-20} \mathrm{~g}$, from which in turn a mean particle diameter of $d_{P}=5.4 \mathrm{~nm}$ can be determined, assuming a density for the silica particles of $\rho=2.65 \mathrm{~g} \mathrm{~cm}^{-3}$.

Fig. 4 summarizes the measured mean particle mass as a function of the flow coordinate (upper part) and as a function of the precursor concentration (lower part) for the $\mathrm{SiH}_{4}$-doped flames. The pressure was kept constant at $p=30 \mathrm{mbar}$, the velocity of the unburnt gases was $v_{u}=1.32 \mathrm{~m} \mathrm{~s}^{-1}, \mathrm{H}_{2} / \mathrm{O}_{2}=1.69$, and the ratio of the inert gas to the fuel gases $\mathrm{Ar} /\left(\mathrm{H}_{2}+\mathrm{O}_{2}\right)$ $=1.36$ and 1.04, respectively. As can be seen in the upper part of Fig. 4, particles formed in the hotter flame (open triangles) show an evolution of the mean particle mass from about $m_{P}=10 \cdot 10^{-20} \mathrm{~g}$ at $\xi=20 \mathrm{~mm}$ to about $m_{P}=22 \cdot 10^{-20} \mathrm{~g}$ at $\xi=70 \mathrm{~mm}$, whereas the par-
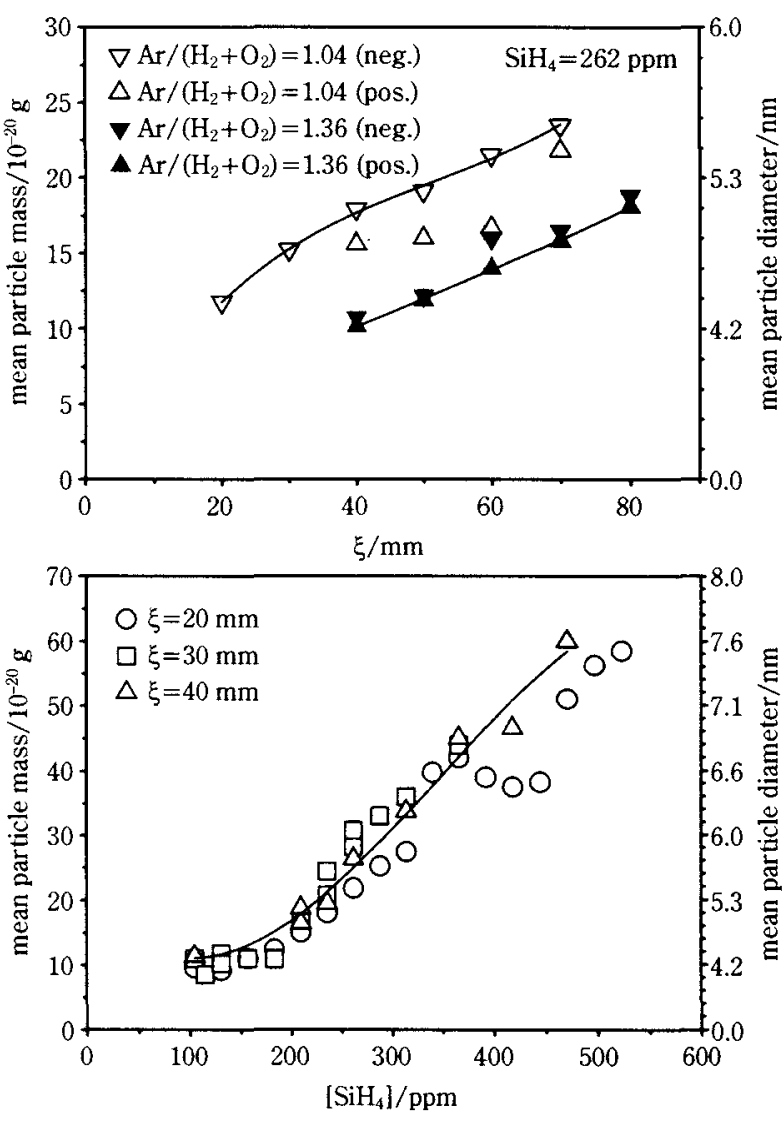

Fig. 4 Particle size measurements of $\mathrm{SiO}_{2}$ particles as a function of the flow coordinate $\xi$ (upper part) and precursor concentration (lower part). Flame conditions: $\mathrm{H}_{2} / \mathrm{O}_{2}=1.69$, $p=30 \mathrm{mbar}, v_{\mathrm{u}}=1.32 \mathrm{~m} \mathrm{~s}^{-1}$.

ticles generated in the cooler flame (closed symbols) are smaller by a factor of about 1.7 . The mean particle mass was measured at three different positions in the flame as a function of initial precursor concentration, see lower part of Fig. 4. The dependence of mean particle mass on the sampling position is not very distinct in the range $20 \mathrm{~mm} \leq \xi \leq 40 \mathrm{~mm}$, whereas it is obvious that the mean mass increases with increasing initial concentration of the dopant.

In the next series of experiments, the PMS was applied to particles generated by a spark generator using three different types of electrode materials. Conventional spark plug material was operated at atmospheric pressure, a silver electrode was used at $400 \mathrm{mbar}$, and for glassy carbon (Sigradur-G), the pressure was held between 20 and 30 mbar. Fig. 5 shows results of the measured probability density of the particle diameter obtained from the three materials at a spark frequency of $f=1000 \mathrm{~Hz}$. All particles show a very narrow size distribution with mean particle sizes between 2 and $4 \mathrm{~nm}$. Fig. 6 shows the dependence of particle size on both the spark frequency and the ignition voltage. The mean particle diameter 


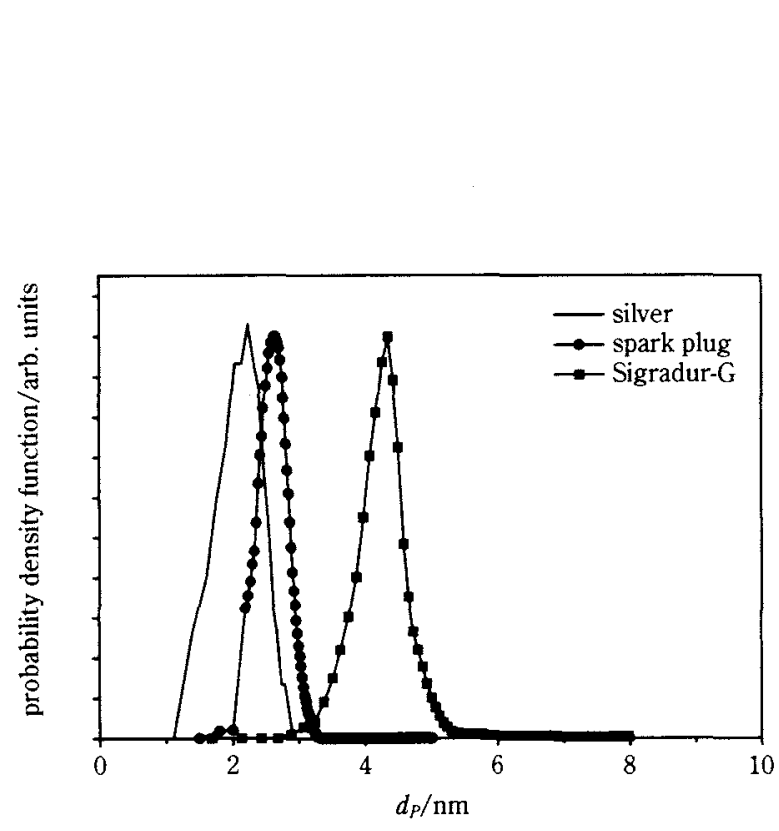

Fig. 5 Diameter-PDF of silver, glassy carbon (Sigradur-G), and conventional spark plug material. Spark frequency: $f=1000 \mathrm{~Hz}$.
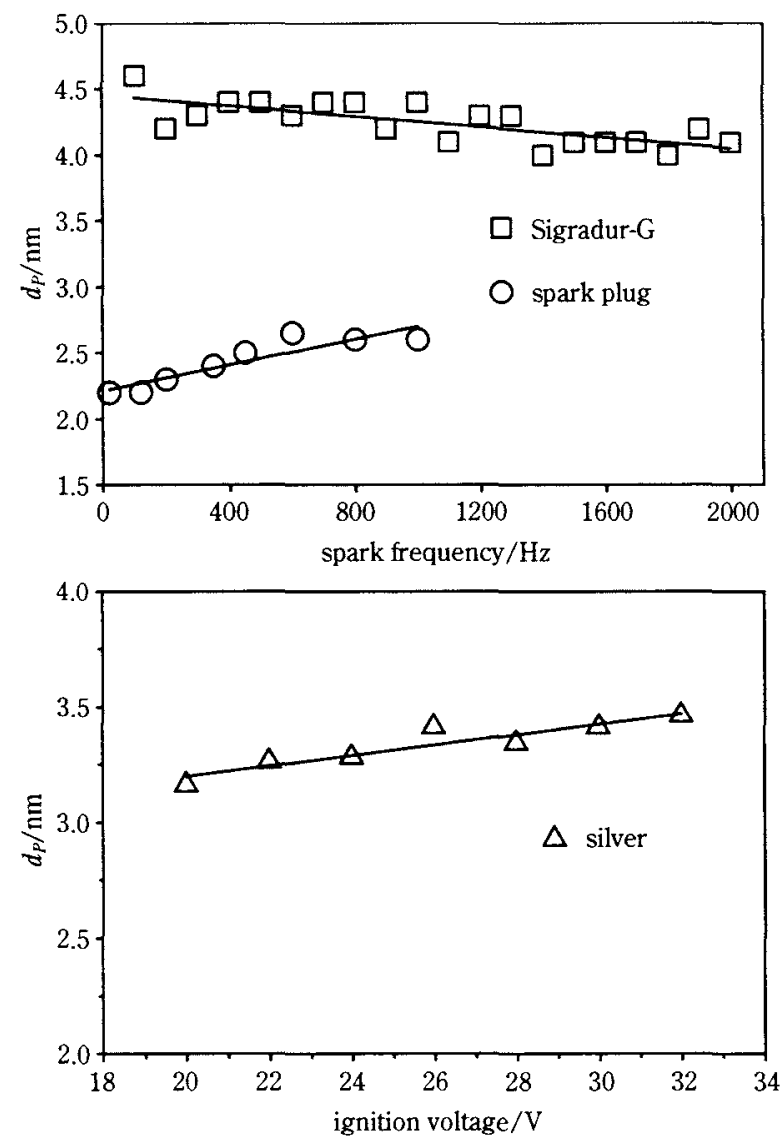

Fig. 6 Influence of spark frequency on the mean particle diameter of Sigradur-G and spark plug material (upper part) and of the ignition voltage on the mean diameter of silver (lower part).

increases slightly from $2.2 \mathrm{~nm}$ to $2.6 \mathrm{~nm}$ with spark frequency for normal spark material (open circles), and decreases from $4.5 \mathrm{~nm}$ to $4.2 \mathrm{~nm}$ for Sigradur-G, see upper part of Fig. 6. For silver electrodes, the frequency has almost no influence on the particle size. On the other hand, the ignition voltage and thus the spark energy is the main parameter determining the

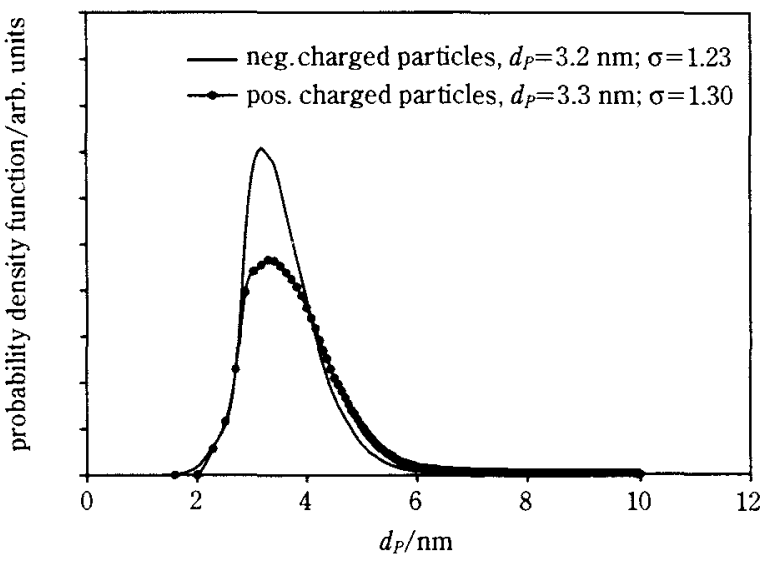

Fig. 7 Diameter-PDF of positively and negatively charged soot particles produced in a microwave reactor.

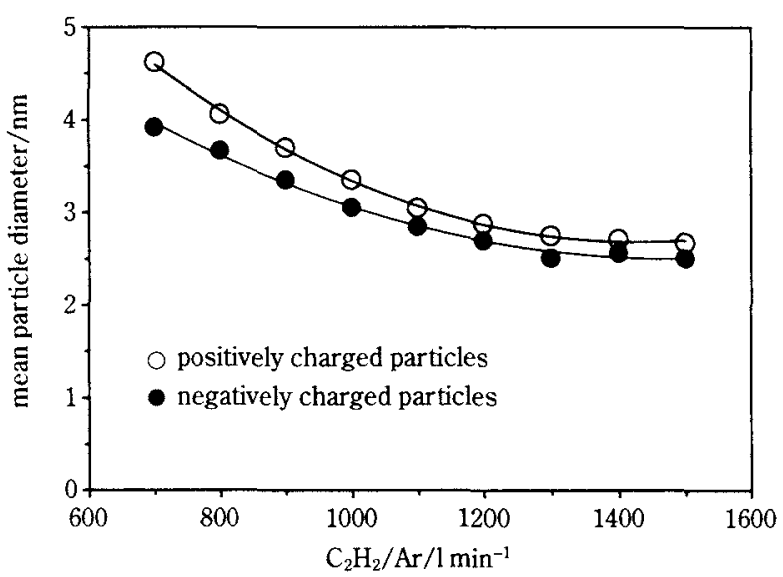

Fig. 8 Influence of the carrier gas flow on the mean particle diameter of soot from the microwave reactor.

mean size of the silver particles, see lower part of Fig. 6. The mean size shows linear behavior, increasing from 3.1 to $3.4 \mathrm{~nm}$.

Next, soot particles formed during pyrolysis of a $\mathrm{C}_{2} \mathrm{H}_{2} / \mathrm{Ar}$ mixture in a microwave reactor were investigated. Fig. 7 shows the measured probability density function of the particle diameter for negatively and positively charged particles. According to Fig. 7, the mean diameter for both polarities is almost the same, but the standard deviation is slightly different. An increase in the flow velocity of the $\mathrm{C}_{2} \mathrm{H}_{2} / \mathrm{Ar}$ mixture through the microwave reactor causes a decrease in the residence time of the particles in the reaction zone. Fig. 8 shows the expected trends of decreasing particle diameter with increasing mixture volume flow.

The last series of experiments was conducted on particles generated in sooting $\mathrm{C}_{2} \mathrm{H}_{2} / \mathrm{O}_{2} / \mathrm{Ar}$ flames. The aim was to compare Faraday-Cup measurements with those performed with an external dynode and the secondary electron multiplier. The main parameter for signal amplification is the voltage applied to 


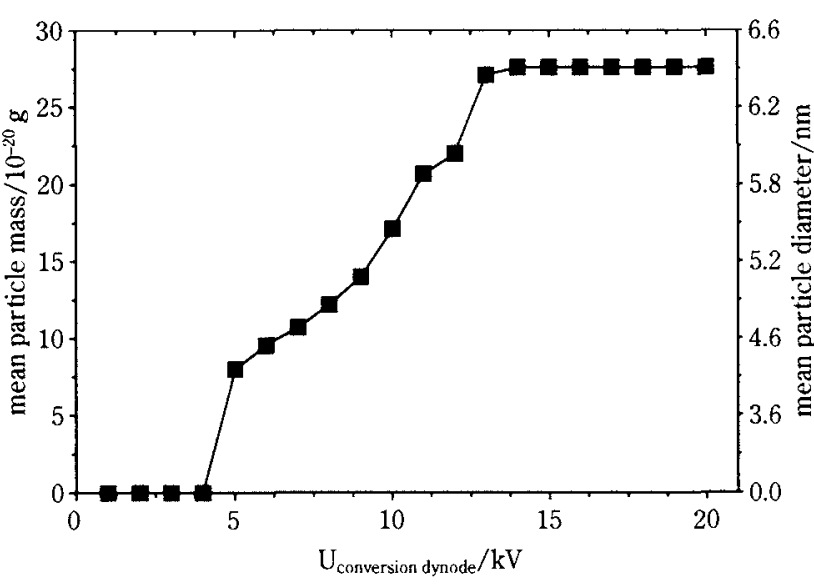

Fig. 9 Measured apparent mean mass and diameter of soot particles originating from a $\mathrm{C}_{2} \mathrm{H}_{2} / \mathrm{O}_{2} / \mathrm{Ar}$ flame $(\mathrm{C} / \mathrm{O}=1.2$, $p=30 \mathrm{mbar}, \xi=30 \mathrm{~mm}$ ) as a function of the conversion dynode voltage.

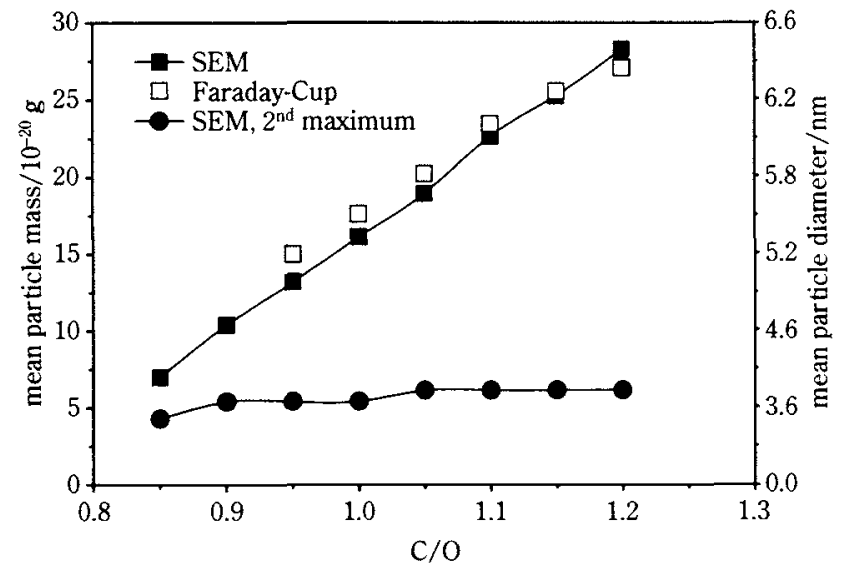

Fig. 10 Mean mass and diameter of negatively charged soot particles generated in $\mathrm{C}_{2} \mathrm{H}_{2} / \mathrm{O}_{2} / \mathrm{Ar}$ flames with various $\mathrm{C} / \mathrm{O}$ ratios measured with the SEM as well as with Faraday-Cup.

the external dynode, which is proportional to the strength of the electric field between the dynode and the multiplier. The acceleration of the particles in the electric field must be high enough to produce secondary electrons by impaction on the surface of the dynode. Initially, the influence of the dynode voltage on the mean properties of soot particles was investigated. A flame burning with a $\mathrm{C} / \mathrm{O}$ ratio of 1.2 was employed, and particles were extracted from a flow coordinate of $\xi=30 \mathrm{~mm}$ above the burner head. As shown in Fig. 9, the apparent mean particle mass first increases with increasing voltage and then remains constant at over $13 \mathrm{kV}$. The voltage-independent size of $d_{P}=6.41 \mathrm{~nm}$ is in agreement with the Faraday-Cup measurement of $d_{P}=6.36 \mathrm{~nm}$. From these experiments, the minimum external dynode voltage was determined, which yields a particle mass that is independent of the acceleration voltage. Fig. 10 shows the mean mass and diameter of negatively charged

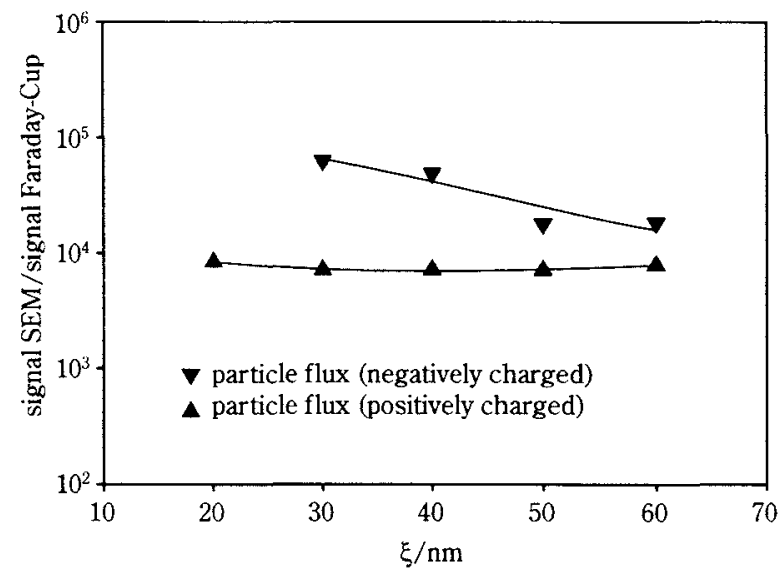

Fig. 11 Relative signal gain ( $\mathrm{I}_{\mathrm{P}-\mathrm{SEM}} / \mathrm{I}_{\mathrm{P} \text {-Faraday-Cup }}$ ) as a function of the flow coordinate.

soot particles, generated in $\mathrm{C}_{2} \mathrm{H}_{2} / \mathrm{O}_{2} / \mathrm{Ar}$ flames with $\mathrm{C} / \mathrm{O}$ ratios of $0.85 \leq \mathrm{C} / \mathrm{O} \leq 1.2$. The flames were burned at $30 \mathrm{mbar}$ and the samples were taken at a fixed flow coordinate of $\xi=30 \mathrm{~mm}$. The secondary electron multiplier (closed symbols) and the FaradayCup (open symbols) measurements show no significant differences in size for $\mathrm{C} / \mathrm{O}>0.95$, indicating the correctness of the multiplier transfer function. Due to the much larger signal of the multiplier compared with the Faraday-Cup measurements, it was also possible to detect soot particles for extremely mild sooting conditions. Furthermore, an additional particle fraction of $d_{P}=3.6 \mathrm{~nm}$ was identified, which could not be resolved by the Faraday-Cup device. The size of this particle class seems to be independent of the burning conditions of the sooting flames. The overall gain in the particle current obtained by the multiplier technique is summarized in Fig. 11. The particle flux measured with the SEM is related to the particle flux measured with the Faraday-Cup as a function of the flow coordinate $\xi$. The signal gain for positively charged particles is about $10^{4}$, whereas negatively charged particles lead to a multiplier current which is always larger than $10^{4}$.

\section{Conclusion}

The particle mass spectrometer (PMS) has been successfully applied to four different aerosols generated under different conditions in various pressure ranges. Soot as well as ceramic or metallic particles were generated in low-pressure flames, microwave plasma, and electric sparks, yielding particles in the size range of 1 to $10 \mathrm{~nm}$. The only conditions of operation are that the process is stationary over a sample time of about two minutes and that the process results 
in charged particles. The use of a secondary electron multiplier with an external dynode as a preamplifier leads to a clear increase in the sensitivity of the PMS. As expected, the particle diameter, being the most important parameter, is not affected by the multiplier. The signal could be increased by a factor of $10^{4}$. It would be possible to additionally improve the sensitivity by further lowering the pressure in the detection chamber, which has a twofold influence. On the one hand, the signal-to-noise-ratio will increase due to the reduced gas in the vacuum chamber, and on the other hand, the reduced pressure will allow the use of a higher voltage applied to the conversion dynode which would lead to a higher degree of conversion, and hence an additional signal gain.

\section{Acknowledgment}

Financial support from the German National Science Foundation (DFG) is gratefully acknowledged.

\section{Nomenclature}

$$
b_{C} \quad \text { : deflection capacitor width }
$$

$d_{P} \quad$ : particle diameter

$e \quad$ : elementary charge

$E \quad$ : kinetic energy

$I_{P} \quad$ : particle current

$l_{C} \quad:$ deflection capacitor length

$l_{G} \quad$ : distance between repelling grids

$l_{P} \quad$ : length of particle package

$m_{P} \quad$ : particle mass

$t \quad$ : time

$U_{C} \quad$ : deflection voltage

$v_{P} \quad$ : particle velocity

$x_{P}:$ : coordinate of a particle

$\dot{x}_{P} \quad$ : particle velocity in $\mathrm{x}$-direction

$y_{P} \quad$ : y coordinate of a particle
$\dot{y}_{P} \quad$ : particle velocity in y-direction

$\left[\mathrm{m} \mathrm{s}^{-1}\right]$

$z \quad$ : number of elementary charges on a particle

$\xi \quad$ : flow coordinate

\section{References}

1) Chen, Y.; Glumac, N.; Kear, B.H.; and Skandan, G.: High rate synthesis of nanophase materials. Nanostructured Materials 9, 1997, pp. 101-104

2) Ulrich, G.D.: Flame synthesis of fine particles. $C \& E N$ 62,1984, pp. $22-29$

3) Lindackers, D.; Janzen, C.; Rellinghaus, B.; Wassermann, E.F.; and Roth, P.: Synthesis of $\mathrm{Al}_{2} \mathrm{O}_{3}$ and $\mathrm{SnO}_{2}$ particles by oxidation of metalorganic precursors in premixed $\mathrm{H}_{2} / \mathrm{O}_{2} / \mathrm{Ar}$ low-pressure flames. Nanostructured Materials 10, 1998, pp. 1247-1270

4) Kobata, A; Kusababe, K.; and Morooka, S.: Growth and transformation of $\mathrm{TiO}_{2}$ crystallites in aerosol reactor. AIChE Journal 37, 1991, pp. 347-356

5) Kusters, K.A. and Pratsinis, S.E.: Strategies for control of ceramic powder synthesis by gas-to-particle conversion. Powder Technology 82, 1995, pp. 79-91

6) Whitby, E. and Hoshino, M.: Development of a lowpressure aerosol sampler. Rev. Sci. Instrum. 66, 1995, pp. 3955-3965

7) Roth, P. and Hospital, A: Mass growth and coagulation of soot particles in low-pressure flames. $24^{\text {th }}$ Symposium (International) on Combustion. The Combustion Institute, Pittsburgh, 1992, pp. 981-989

8) Ziemann, P.J.; Liu, P; Rao, N.P; Kittelson, D.B.; McMurry, P.H.: Particle Beam Mass Spectrometry of Submicron Particles Charged to Saturation in an Electron Beam. J. Aerosol Sci., 26, 1995, pp. 745-756

9) Roth, P. and Hosptial, A.: Design and test of a particle mass spectrometer (PMS). J. Aerosol Sci. 25, 1994, pp. $61-73$

10) Lindackers, D.; Strecker, M.G.D.; Roth, P.; Janzen, C.; Pratsinis, S.E.: Formation and Growth of $\mathrm{SiO}_{2}$ Particles in Low-Pressure $\mathrm{H}_{2} / \mathrm{O}_{2} / \mathrm{Ar}$ Flames Doped with $\mathrm{SiH}_{4}$. Combust. Sci. Technol., 123, 1997, pp. 287-315 


\section{Author's short biography}

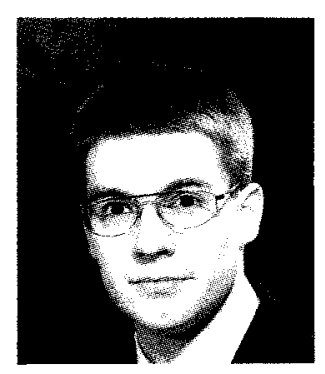

\section{Janzen}

Christian Janzen graduated in Mechanical Engineering at the University of Duisburg, Germany, in 1996. He is currently working on a $\mathrm{Ph} . \mathrm{D}$. project in the Sonderforschungsbreich 445 . The subject of his thesis deals with formation and characterization of nanoparticles from low pressure flame reactors.

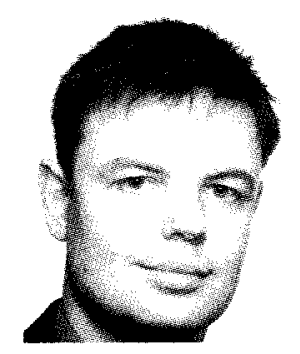

\section{M.G.D. Strecker}

Marc G. D. Strecker received is Diploma in Mechanical Engineering from the University of Duisburg, Germany, in 1991. He was working on a Ph.D. project on nanoparticle generation and measuring methods in low pressure reactors in the DFG Schwerpunktprogramm "Feinste, feste Partikeln" since 1992. Since 1998 he is working as a Software Engineer at the "software design \& management AG".

\section{P. Roth}

Dr. Paul Roth is Professor of Combustion and Gasdynamics and director of the respective Institute at the Gerhard-Mercator-Universität in Duisburg. He received his diploma in mechanical engineering from Technical University in Aachen and got his $\mathrm{Ph}$.D. also from Aachen in thermo- and fluiddynamics. He worked for about 10 years as a research assistant and later as a senior scientist at the German Aerospace Research Center (DLR) and moved there after to University of Duisburg. He was for about 15 years speaker of the Aerosol Research Program at Duisburg supported by the German Science Foundation. He is currently heading a new long term program on "Nanoparticles from the gasphase" (SFB 445). He has authored more than 250 articles on reaction kinetics, combustion, aerosols, gasdynamics, engine combustion, and on gas-to-particle conversion processes. Prof. Roth is an editorial board member of the J. Aerosol Science and of the Shock Wave Journal. 